KUNS-1945

OU-HET-502/2004

\title{
Higgs mass in the gauge-Higgs unification
}

\author{
Naoyuki Haba ${ }^{(a), *}$ Kazunori Takenaga $^{(b), ~} \dagger \operatorname{Toshifumi~Yamashita~}^{(c), ~} \ddagger$ \\ (a) Institute of Theoretical Physics, University of Tokushima, Tokushima 770-8502, \\ Japan \\ (b) Department of Physics, Osaka University, Toyonaka, Osaka 560-0043, Japan \\ ${ }^{(c)}$ Department of Physics, Kyoto University, Kyoto, 606-8502, Japan
}

\begin{abstract}
The gauge-Higgs unification theory identifies the zero mode of the extra dimensional component of the gauge field as the usual Higgs doublet. Since this degree of freedom is the Wilson line phase, the Higgs does not have the mass term nor quartic coupling at the tree level. Through quantum corrections, the Higgs can take a vacuum expectation value, and its mass is induced. The radiatively induced mass tends to be small, although it can be lifted to $\mathcal{O}(100)$ $\mathrm{GeV}$ by introducing the $\mathcal{O}(10)$ numbers of bulk fields. Perturbation theory becomes unreliable when a large number of bulk fields are introduced. We reanalyze the Higgs mass based on useful expansion formulae for the effective potential and find that even a small number of bulk field can have the suitable heavy Higgs mass. We show that a small (large) number of bulk fields are enough (needed) when the SUSY breaking mass is large (small). We also study the case of introducing the soft SUSY breaking scalar masses in addition to the Scherk-Schwarz SUSY breaking and obtain the heavy Higgs mass due to the effect of the scalar mass.
\end{abstract}

\footnotetext{
*E-mail: haba@ias.tokushima-u.ac.jp

†E-mail: takenaga@het.phys.sci.osaka-u.ac.jp

${ }^{\ddagger}$ E-mail: yamasita@gauge.scphys.kyoto-u.ac.jp
} 


\section{Introduction}

There are much progress in the higher dimensional gauge theories. One of the most fascinating motivations for the higher dimensional gauge theory is that gauge and Higgs fields can be unified [1]-[13]. The higher dimensional components of gauge fields become scalar fields bellow the compactification scale, and these scalar fields are identified with the Higgs fields in the gauge-Higgs unification theory. In fact, the adjoint Higgs fields can emerge through the $S^{1}$ compactification from 5D theory, while the Higgs doublet fields can appear through the orbifold compactification such as $S^{1} / Z_{2}$.

In order to obtain the Higgs doublets from the gauge fields in higher dimensions, the gauge group must be lager than the standard model (SM) gauge group. The gauge symmetries are reduced by the orbifolding boundary conditions of the extra dimensions and can be broken further by the Hosotani mechanism [2]. The Higgs fields have only finite masses of order the compactification scale because the masses of the Higgs fields are forbidden by the higher dimensional gauge invariance.

In the previous works 11, 12, we have studied the possibility of the dynamical electro-weak symmetry breaking in two gauge-Higgs unified models, $S U(3)_{c} \times S U(3)_{W}$ and $S U(6)$ models. We calculated the one loop effective potential of Higgs doublets and analyze the vacuum structure of the models, and a similar analysis of the 6D gauge-Higgs unification model is studied in Ref. [13]. We found that the introduction of the appropriate numbers and representation of extra bulk fields are required for the desirable symmetry breaking. Since the Higgs is essentially the Wilson line degree of freedom, the mass term nor quartic coupling does not exist in the Higgs potential at the tree level. Through quantum corrections, the Higgs can develop a vacuum expectation value, which means the dynamical electro-weak symmetry breaking is realized and accordingly its mass is induced. The induced Higgs mass tends to be small, less than the weak scale, reflecting the nature of the Coleman-Weinberg mechanism [14.

It is possible to lift the magnitude of the Higgs mass to $\mathcal{O}(100) \mathrm{GeV}$ by introducing the $\mathcal{O}(10)$ numbers of bulk fields in Refs. [11, 12. The perturbation expansion is given

by $g^{2} / 16 \pi^{2} \times$ [bulk fields degrees of freedom] $(g$ : gauge coupling $)$, so that the analysis of the one loop effective potential can not be reliable when there are a large number of bulk fields. Furthermore, it seems artificial to introduce a large number of extra fields.

In this paper we reanalyze and study the Higgs mass analytically, not only numerically in the gauge-Higgs unification. Based on the expansion formulae for the effective potential, we show that a small (large) number of bulk fields are needed when the SUSY breaking mass is large (small). We find that even a small number of bulk field can make the Higgs mass suitably heavy. The expression for the Higgs mass is obtained by using the formula, which makes it clear that what mainly controls the magnitude of the Higgs mass. The analyses made in this paper by using the 
expansion formula are applicable to the bulk field with an arbitrary representation under an arbitrary gauge group.

We also study the case that the soft SUSY breaking scalar masses exist in addition to the Scherk-Schwarz (SS) SUSY breaking [15]-[19]. The soft scalar masse also plays the role to lift the Higgs mass. And we show that also in this case a small $(\mathcal{O}(1))$ number of extra bulk fields can realize the suitable electro-weak symmetry breaking and the Higgs mass of $\mathcal{O}(100) \mathrm{GeV}$.

This paper is organized as follows. In section 2, we briefly overview the previous works. In section 3 we will present useful expansion formulae for the effective potential, and by using them, we study the Higgs mass in the gauge-Higgs unified models. In section 4, we also study the Higgs mass for the case of existing soft SUSY breaking scalar masses. Section 5 devotes summary and discussion.

\section{Gauge-Higgs unification}

In this section we give a brief review of the dynamical electro-weak symmetry breaking in the $S U(3)_{c} \times S U(3)_{W}$ and $S U(6)$ models based on Refs. 11, 12. In the gaugeHiggs unification models, the 5 th dimensional coordinate is compactified on an $S^{1} / Z_{2}$ orbifold. The Higgs doublets are identified as the zero modes of the extra dimensional component of the 5D gauge field, $A_{5}$. We check whether the dynamical electro-weak symmetry breaking is possible or not by calculating one loop effective potential of the Higgs doublets.

Denoting $y$ as the coordinate of the 5th dimension, parity operator, $P\left(P^{\prime}\right)$ are defined according to the $Z_{2}$ transformation, $y \rightarrow-y(\pi R-y \rightarrow \pi R+y)$. In the $S U(3)_{c} \times S U(3)_{W}$ model [4, [5, 6], we take $P=P^{\prime}=\operatorname{diag}(1,-1,-1)\left(P=P^{\prime}=I\right)$ in the base of $S U(3)_{W}\left(S U(3)_{c}\right)$. Then, there appears Higgs doublet as the zero mode of $A_{5} \S$,

$$
H=\sqrt{2 \pi R} A_{5} .
$$

The 4D gauge coupling constant is defined as $g_{4}=g / \sqrt{2 \pi R}$. The vacuum expectation value (VEV) of $A_{5}$ is parameterized by $a /(2 g R) \mathbf{E}_{3}$, where $\mathbf{E}_{3}$ is the $3 \times 3$ matrix having 1 at $(1,3)$ and $(3,1)$ elements, while the other elements being zero 11,12 . The relation between the $\mathrm{VEV}$ and electro-weak scale is given by

$$
\sqrt{2 \pi R}\left\langle A_{5}^{4}\right\rangle=\frac{a_{0}}{g_{4} R}=v \sim 246 \mathrm{GeV} .
$$

Here the component gauge field $A_{5}^{4}$ is defined by $A_{5}=\sum_{a} A_{5}^{a} T^{a}$ through the generators $T^{a}$, where $T^{4}=\frac{1}{2} \mathbf{E}_{3}$. The compactification scale must be above the weak

\footnotetext{
$\S$ Taking account of the scalar degrees of freedom in the gauge super multiplet, we can easily show that there appear two Higgs doublets in the SUSY theory.

"We should take $g_{4} \gtrsim 1$ for the wall-localized kinetic terms being the main part of the MSSM kinetic terms 11]. Hence, we take $g_{4}=\mathcal{O}(1)$ in this paper.
} 
scale, and when we take it as a few TeV, for examples, $a_{0}$ should be a parameter of $\mathcal{O}\left(10^{-1 \sim-2}\right)$.

Let us study SUSY theory with SS SUSY breaking. We define

$$
\begin{aligned}
J^{(+)}[a, \beta, n] & \equiv \frac{1}{n^{5}}(1-\cos (2 \pi n \beta)) \cos (\pi n a), \\
J^{(-)}[a, \beta, n] & \equiv \frac{1}{n^{5}}(1-\cos (2 \pi n \beta)) \cos (\pi n(a-1)),
\end{aligned}
$$

where $\beta(0 \leq \beta \leq 0.5)$ parameterizes the magnitude of the SS SUSY breaking. Then, the soft mass parameters become $\mathcal{O}(\beta / R)[11$, 12. The contribution of the gauge multiplet to the effective potential is written as

$$
V_{\text {eff }}^{\text {gauge }}=-2 C \sum_{n=1}^{\infty}\left(J^{(+)}[2 a, \beta, n]+2 J^{(+)}[a, \beta, n]\right),
$$

where $C \equiv 3 /\left(64 \pi^{7} R^{5}\right)$. The VEV of $\sigma$, which forms the real part of scalar component of $N=1$ chiral multiplet at low-energies, becomes zero by calculation of the effective potential for $\langle\sigma\rangle\left[20\right.$. The minimum of the effective potential (3) is located at $a_{0}=$ $1(\bmod 2)$, which means that the suitable electro-weak scale VEV, $(0<) a_{0} \ll$ 1 and electro-weak symmetry breaking are not realized. Thus, for the desirable dynamical electro-weak symmetry breaking, one needs to introduce the extra bulk fields, which are $N_{f n d}^{( \pm)}$and $N_{a d j}^{( \pm)}$. species of hypermultiplets of fundamental and adjoint representations, respectively. Here the index, $( \pm)$, denotes the sign of the intrinsic parity of $P P^{\prime}$ defined in Refs. [11, 12].

The effective potential from the bulk fields is given by

$$
\begin{array}{rl}
V_{\text {eff }}^{\text {matter }}=2 & C \sum_{n=1}^{\infty}\left\{N_{a d j .}^{(+)}\left(J^{(+)}[2 a, \beta, n]+2 J^{(+)}[a, \beta, n]\right)\right. \\
& +N_{a d j .}^{(-)}\left(J^{(-)}[2 a, \beta, n]+2 J^{(-)}[a, \beta, n]\right) \\
& \left.+N_{\text {fnd. }}^{(+)} J^{(+)}[a, \beta, n]+N_{\text {fnd. }}^{(-)} J^{(-)}[a, \beta, n]\right\} .
\end{array}
$$

Reference [11] shows one example, $N_{a d j .}^{(+)}=N_{a d j .}^{(-)}=2, N_{\text {fnd. }}^{(-)}=4, N_{\text {fnd. }}^{(+)}=0$ with $\beta=0.1$ and $R^{-1}$ of order a few $\mathrm{TeV}$, in which the suitable electro-weak symmetry breaking is realized by the small VEV,$a_{0}=0.047$. The Higgs mass is calculate by the second derivative of the effective potential, $V_{\text {eff }} \equiv C \bar{V}_{\text {eff }}=V_{\text {eff }}^{\text {gauge }}+V_{\text {eff }}^{\text {matter }}$ with respect to $a$ at the minimum, $a=a_{0}$,

$$
m_{H} \sim \frac{\sqrt{3}}{4 \pi^{3}}\left(\frac{\partial^{2} \bar{V}_{\text {eff }}}{\partial a^{2}}\right)_{a=a_{0}}^{1 / 2} \times \frac{v g_{4}^{2}}{a_{0}}
$$


where we have used (2). In this case Higgs mass is calculated as $\|$

$$
m_{H}^{2} \sim\left(\frac{0.025 g_{4}}{R}\right)^{2} \sim\left(118 g_{4}^{2} \mathrm{GeV}\right)^{2},
$$

where $g_{4}=\mathcal{O}(1)$, as explained above. The Higgs mass is likely to be smaller than the weak scale, $246 \mathrm{GeV}$ (Eq.(2) ) since it is zero at the tree level and is induced through the radiative corrections (Coleman-Weinberg mechanism).

As for the $S U(6) \operatorname{model}[5$, [6], we take the parities, $P=\operatorname{diag}(1,1,1,1,-1,-1)$ and $P^{\prime}=\operatorname{diag}(1,-1,-1,-1,-1,-1)$, which induces Higgs doublet in $A_{5}$ as the zero mode. The VEV of $A_{5}$ is written as $a /(2 g R) \mathbf{E}_{6}$, where $\mathbf{E}_{6}$ is the $6 \times 6$ matrix having 1 at $(1,6)$ and $(6,1)$ elements while the other elements being zero 11, 12]. The gauge part of the effective potential is given by

$$
V_{\text {eff }}^{\text {gauge }}=-2 C \sum_{n=1}^{\infty}\left(J^{(+)}[2 a, \beta, n]+2 J^{(+)}[a, \beta, n]+6 J^{(-)}[a, \beta, n]\right) .
$$

As in the $S U(3)_{c} \times S U(3)_{W}$ model, the suitable symmetry breaking can not be realized only by the gauge sector. This situation can be changed by introducing the extra bulk fields, which induce the effective potential,

$$
\begin{aligned}
& V_{\text {eff }}^{\text {matter }}=2 C \sum_{n=1}^{\infty}\left\{N_{a d j .}^{(+)}\left(J^{(+)}[2 a, \beta, n]+2 J^{(+)}[a, \beta, n]+6 J^{(-)}[a, \beta, n]\right)\right. \\
&+ N_{a d j .}^{(-)}\left(J^{(-)}[2 a, \beta, n]+2 J^{(-)}[a, \beta, n]+6 J^{(+)}[a, \beta, n]\right) \\
&\left.+N_{\text {fnd. }}^{(+)} J^{(+)}[a, \beta, n]+N_{\text {fnd. }}^{(-)} J^{(-)}[a, \beta, n]\right\} .
\end{aligned}
$$

We show one example of the suitable symmetry breaking in Ref.[11], which is the case of $N_{a d j .}^{(+)}=2, N_{\text {fnd. }}^{(-)}=10, N_{\text {adj. }}^{(-)}=N_{\text {fnd. }}^{(+)}=0$ with $\beta=0.1$ and $R^{-1}$ of order a few $\mathrm{TeV}$. In this case, the minimum exists at $a_{0}=0.047$, and the Higgs mass squared is calculated as

$$
m_{H}^{2} \sim\left(\frac{0.024 g_{4}}{R}\right)^{2} \sim\left(120 g_{4}^{2} \mathrm{GeV}\right)^{2} .
$$

In the above two examples $\mathcal{O}(10)$ numbers of bulk fields are required for the suitable symmetry breaking and Higgs mass. Naively, this situation seems inevitable in the gauge-Higgs unification theory since Higgs doublets are originally Wilson line phases and do not have the quartic couplings nor mass terms in the tree level.

In the next section we obtain the effective mass term and quartic coupling by expanding the cosine functions with respect to $a$ in the effective potential and study the condition for the suitable symmetry breaking and Higgs mass. We will check whether a large numbers of extra fields are really needed or not.

\footnotetext{
"In Reference [1], we calculated it by using approximation formulae, and got slightly different value.
} 


\section{Higgs mass}

The Higgs mass is defined by the 2nd derivative of the effective potential. Here we concentrate on the mass term and quartic coupling in the radiatively induced effective potential. We comment on higher order terms in the last section. The effective potentials in the previous section are written by the linear combinations of $J^{( \pm)}[a, \beta, n]$ and $J^{( \pm)}[2 a, \beta, n]$. Effective potentials are generally the linear combination of $J^{( \pm)}[m a, \beta, n],(m$ : integer), (see for examples, Refs. 11, 12, 21]). Here we study the contribution from the fundamental representation bulk fields for simplicity. Although the gauge sector and adjoint representation bulk fields also induce $J^{( \pm)}[2 a, \beta, n]$ terms (higher representations can induce $J^{( \pm)}[m a, \beta, n]$ in general), these contributions can be incorporated straightforwardly.

By using approximations for small $x$, we obtain, up to $\mathcal{O}\left(x^{8}\right)$, that

$$
\begin{aligned}
& \sum_{n=1}^{\infty} \frac{\cos (n x)}{n^{5}} \sim \zeta_{R}(5)-\frac{\zeta_{R}(3)}{2} x^{2}-\frac{x^{4}}{48} \ln \left(x^{2}\right)+\frac{25}{288} x^{4}+\frac{x^{6}}{8640}+\frac{x^{8}}{4838400}, \\
& \sum_{n=1}^{\infty} \frac{\cos (n(x-\pi))}{n^{5}} \sim-\frac{15}{16} \zeta_{R}(5)+\frac{3}{8} \zeta_{R}(3) x^{2}-\frac{x^{4}}{24} \ln 2+\frac{x^{6}}{2880}+\frac{x^{8}}{322560} .
\end{aligned}
$$

Let us note that these formulae are also applicable to the bulk field with the higher representations under the gauge group. Then, we can show that

$$
\begin{aligned}
& \sum_{n=1}^{\infty} J^{(+)}\left[\frac{a}{\pi}, \frac{\beta}{\pi}, n\right] \sim \frac{a^{2}}{288}\left(25 a^{2}-432 \beta^{2}-6 a^{2} \ln \left(\frac{a^{2}}{4 \beta^{2}}\right)+144 \beta^{2} \ln \left(4 \beta^{2}\right)\right) \\
& \sum_{n=1}^{\infty} J^{(-)}\left[\frac{a}{\pi}, \frac{\beta}{\pi}, n\right] \sim-\frac{\beta^{2}}{48}\left(a^{4}-48 a^{2} \ln 2\right) .
\end{aligned}
$$

for $a \ll \beta^{* *}$. Then, the coefficients of $a^{2}$ and $a^{4}$ in Eq.(12) are roughly given by

$$
-\frac{\beta^{2}}{288}\left(432-144 \ln \left(4 \beta^{2}\right)\right) \quad(<0) \text { and } \frac{25-6 \ln \left(a_{0}^{2} / 4 \beta^{2}\right)}{288}(>0) \text {, }
$$

respectively. On the other hand, coefficients of $a^{2}$ and $a^{4}$ in Eq.(13) are

$$
\beta^{2} \ln 2(>0), \quad \text { and } \quad-\beta^{2} / 48(<0),
$$

respectively.

For realizing the suitable heavy Higgs mass, the quartic coupling should be large and positive. On the other hand, the VEV (W and Z boson masses) should be maintained small $\left(a_{0} \ll 1\right)$ comparing to the compactification scale. For this purpose,

${ }^{* *}$ In the usual scenario, $a<\beta$ should be satisfied since the SUSY breaking mass, $\mathcal{O}(\beta / R)$ must be larger than the electro-weak scale, $\mathcal{O}(a / R)$. 
large negative contribution in the first term in Eq.(14) must be almost cancelled by introducing $N_{\text {fnd. }}^{(-)}=\mathcal{O}\left(\ln 4 \beta^{2}\right)$ numbers bulk fields acting on the first term in Eq.(15)). This means that the less (more) bulk fields are needed when $\beta$ becomes large (small). Eqs.(14) and (15) shows that even in the case of this cancellation, the coefficient of $a^{4}$ is still positive and large enough when $a_{0} \ll \beta$. Thus, the heaviness of Higgs mass is mainly controlled by the factor $-\ln \left(a_{0}^{2} / \beta^{2}\right)$ in the effective quartic coupling, which implies that the smaller (larger) $a_{0}^{2} / \beta^{2}$ becomes, the larger (smaller) the Higgs mass becomes. A typical numerical example for realizing this in the $S U(3)_{c} \times S U(3)_{W}$ model is given in the table.

\begin{tabular}{|cccc|ccc|}
\hline$N_{\text {adj. }}^{(+)}$ & $N_{\text {adj. }}^{(-)}$ & $N_{\text {fnd. }}^{(+)}$ & $N_{\text {fnd. }}^{(-)}$ & $\beta$ & $a_{0}$ & $m_{H} / g_{4}^{2}(\mathrm{GeV})$ \\
\hline \hline 2 & 2 & 0 & 2 & 0.10 & 0.0891 & 95 \\
\hline 2 & 2 & 0 & 2 & 0.13 & 0.0574 & 117 \\
\hline 2 & 2 & 0 & 2 & 0.14 & 0.0379 & 130 \\
\hline
\end{tabular}

The observation given above becomes clearer if we apply (12) and (13) to the effective potential, $V_{\text {eff }}$ and take the order up to $O\left(x^{4}\right)$. Then, the Higgs mass for the $S U(3)_{c} \times$ $S U(3)_{W}$ model is calculated as

$$
\frac{m_{H}}{g_{4}^{2}} \simeq v \frac{\sqrt{3}}{4 \pi} \sqrt{4 B \ln \left(\frac{a_{0}^{2}}{4 \beta^{2}}\right)+\text { const. }}
$$

where

$$
B \equiv \frac{-1}{24}\left(18\left(N_{\text {adj. }}^{(+)}-1\right)+N_{\text {fnd. }}^{(+)}\right),
$$

and the constant term depends on $\beta$ and the number of flavors. Equation (17) shows that a few adjoint bulk fields are enough and essential for the large quartic coupling. The contribution from the adjoint bulk field overcome the loop factor $\sim 1 / 4 \pi$ to enhance the magnitude of the Higgs mass. Let us note that the dependence of the Higgs mass on the supersymmetry breaking parameter is logarithmic, as expected.

If we tune the values of $\beta$ to $\beta_{c} \simeq 0.14865 \cdots$ at which the coefficient of $a^{2}$ vanishes at one-loop level, the smaller VEV $a_{0}$ is realized within the validity of perturbation theory, and the scale $R$ should be smaller due to Eq.(22). In this sence two examples below are different theories from each other, since they should have different initial setup of the value, $R$. The magnitude of the Higgs mass is enhanced because of the large $\ln \left(a_{0}^{2} / \beta^{2}\right)$ as shown in the table for the $S U(3)_{c} \times S U(3)_{W}$ model with $\left(N_{\text {adj. }}^{(+)}, N_{\text {adj. }}^{(-)}, N_{\text {fnd. }}^{(+)}, N_{\text {fnd. }}^{(-)}\right)=(2,2,0,2)^{\dagger \dagger}$.

\begin{tabular}{|cccc|ccc|}
\hline$N_{\text {adj. }}^{(+)}$ & $N_{\text {adj. }}^{(-)}$ & $N_{\text {fnd. }}^{(+)}$ & $N_{\text {fnd. }}^{(-)}$ & $\beta$ & $a_{0}$ & $m_{H} / g_{4}^{2}(\mathrm{GeV})$ \\
\hline \hline 2 & 2 & 0 & 2 & 0.1486 & 0.0023 & 191 \\
\hline 2 & 2 & 0 & 2 & 0.14865 & 0.0009 & 208 \\
\hline
\end{tabular}

\footnotetext{
${ }^{\dagger}$ Note that too small $a_{0}$ induces a very large log factor that spoils the validity of perturbation theory.
} 
Two loop contributions become dominant in the coefficient of $a^{2}$ if we tune $\beta$ close to $\beta_{c}$. The effect of the two loop, however, is almost absorbed into the values of $\beta$ by adjusting $\beta$ at one-loop level as long as the perturbation is valid. If one chooses $\beta$ such that the coefficient of $a^{2}$ taken account of higher loops almost vanishes, one expects very small values of $a_{0}$, so that the magnitude of the Higgs mass becomes larger than the values obtained in the above table.

Let us comment on the heavy Higgs mass in non-SUSY gauge models. We can see from Eqs. (10) and (11) that it is possible to cancel the $a^{2}$ terms between the matter with even parity and the one with odd parity, keeping the positive and large quartic coupling, by an appropriate choice of the matter content. In this case, we have the similar situation with the SUSY case studied above and expect the desirable size of the Higgs mass. In fact, the non-SUSY model with the appropriate matter content, which realizes the suitable dynamical electro-weak symmetry breaking, is presented in Ref.11].

\section{SUSY gauge-Higgs with bulk mass}

In this section we show another example for realizing the dynamical electro-weak symmetry breaking with the small number of extra bulk fields. We introduce explicit soft SUSY breaking scalar mass in addition to the SS parameter for the bulk superfields ${ }^{\ddagger \ddagger}$. In this paper we do not introduce the soft gaugino masses because the mass terms are odd under the $Z_{2}$ operation.

Let us study the $S U(3)_{c} \times S U(3)_{W}$ model at first. The contribution of the gauge multiplet to the effective potential is the same as Eq.(3). We introduce the soft SUSY breaking mass, $m$ for the bulk hypermultiplets and define a dimensionless parameter, $z \equiv m R(<1)$. We denote

$$
\begin{aligned}
I^{(+)}[a, \beta, z, n] & \equiv \frac{1}{n^{5}}\left(1-\left(1+2 \pi z n+\frac{(2 \pi z n)^{2}}{3}\right) e^{-2 \pi z n} \cos (2 \pi n \beta)\right) \\
& \times \cos (\pi n a), \\
I^{(-)}[a, \beta, z, n] & \equiv \frac{1}{n^{5}}\left(1-\left(1+2 \pi z n+\frac{(2 \pi z n)^{2}}{3}\right) e^{-2 \pi z n} \cos (2 \pi n \beta)\right) \\
& \times \cos (\pi n(a-1)),
\end{aligned}
$$

in which $I^{( \pm)}[a, \beta, z, n]$ is reduced to $J^{( \pm)}[a, \beta, n]$ in the limit of $z \rightarrow 0(m \rightarrow 0)$. The

$\ddagger$ The effective potentials and vacuum structures with soft scalar masses on $S^{1}$ have been studied in Refs. 22, 23]. 
contribution of the matter hypermultiplet to the effective potential is given by

$$
\begin{aligned}
V_{\mathrm{eff}}^{\mathrm{matter}}=2 C \sum_{n=1}^{\infty}\left\{N_{\text {adj. }}^{(+)}\left(I^{(+)}\left[2 a, \beta, z_{\text {adj. }}^{(+)}, n\right]+2 I^{(+)}\left[a, \beta, z_{\text {adj. }}^{(+)}, n\right]\right)\right. \\
+N_{\text {adj. }}^{(-)}\left(I^{(-)}\left[2 a, \beta, z_{\text {adj. }}^{(-)}, n\right]+2 I^{(-)}\left[a, \beta, z_{\text {adj. }}^{(-)}, n\right]\right) \\
\left.+N_{\text {fnd. }}^{(+)} I^{(+)}\left[a, \beta, z_{\text {fnd. }}^{(+)}, n\right]+N_{\text {fnd. }}^{(-)} I^{(-)}\left[a, \beta, z_{\text {fnd. }}^{(-)}, n\right]\right\},
\end{aligned}
$$

where $z_{r e p}^{( \pm)}$. stands for the explicit soft mass defined by $z_{r e p}^{( \pm)} \equiv m_{r e p}^{( \pm)} R(<1)$ for each representation field. Eq.(20) becomes Eq.(4) in the limit of the vanishing soft scalar mass, $m \rightarrow 0$.

We find some examples of extra matter contents and SUSY breaking parameters, for which the suitable VEV and Higgs mass are realized, and we summarize them in the following table.

\begin{tabular}{|c|cccc|ccccc||cc|}
\hline & $N_{\text {adj. }}^{(+)}$ & $N_{\text {adj. }}^{(-)}$ & $N_{\text {fnd. }}^{(+)}$ & $N_{\text {fnd. }}^{(-)}$ & $\beta$ & $z_{\text {adj. }}^{(+)}$ & $z_{\text {adj. }}^{(-)}$ & $z_{\text {fnd. }}^{(+)}$ & $z_{\text {fnd. }}^{(-)}$ & $a_{0}$ & $m_{H} / g_{4}^{2}$ \\
\hline \hline$(1)$ & 2 & 3 & 0 & 4 & 0.05 & 0.01 & 0.01 & - & 0.045 & 0.0040 & 164 \\
\hline$(2)$ & 2 & 4 & 2 & 6 & 0.05 & 0 & 0 & 0.05 & 0.05 & 0.0037 & 176 \\
\hline$(3)$ & 2 & 4 & 0 & 6 & 0.025 & 0.025 & 0.025 & - & 0.025 & 0.0066 & 129 \\
\hline$(4)$ & 2 & 1 & 0 & 2 & 0.1 & 0.1 & 0.1 & - & 1 & 0.0097 & 150 \\
\hline$(5)$ & 1 & 1 & 0 & 2 & 0.01 & 1 & 1 & - & 1 & 0.0196 & 125 \\
\hline$(6)$ & 2 & 2 & 0 & 2 & 0.14 & 0 & 0 & - & 0 & 0.0379 & 130 \\
\hline
\end{tabular}

The Higgs mass $m_{H} / g_{4}^{2}$ is measured in GeV unit. This table shows that even small number of extra bulk fields can realize the suitable dynamical electro-weak symmetry breaking with the heavy Higgs mass. The effect of the bulk masses increases not only the degrees of freedom of parameter space, but also induces a similar effect of large $\beta$, which is necessary for the symmetry breaking with a small number bulk fields, as explained in the last section. We show an example in which one can see the enhancement of the magnitude of the Higgs mass due to the existence of the bulk mass in the table, where the Higgs mass $m_{H} / g_{4}^{2}$ is measured in $\mathrm{GeV}$ unit.

\begin{tabular}{|cccc|ccccc||cc|}
\hline$N_{\text {adj. }}^{(+)}$ & $N_{\text {adj. }}^{(-)}$ & $N_{\text {fnd. }}^{(+)}$ & $N_{\text {fnd. }}^{(-)}$ & $\beta$ & $z_{\text {adj. }}^{(+)}$ & $z_{\text {adj. }}^{(-)}$ & $z_{\text {fnd. }}^{(+)}$ & $z_{\text {fnd. }}^{(-)}$ & $a_{0}$ & $m_{H} / g_{4}^{2}$ \\
\hline \hline 2 & 1 & 0 & 2 & 0.1 & 0 & 0 & - & 0 & 0.2362 & 42 \\
\hline 2 & 1 & 0 & 2 & 0.1 & 0.1 & 0.1 & - & 1 & 0.0097 & 150 \\
\hline
\end{tabular}

Next we study the case of $S U(6)$ model. The contribution of the gauge multiplet to the effective potential is the same as Eq.(7). On the other hand, the contribution 
of the matter hypermultiplet to the effective potential is given by

$$
\begin{aligned}
V_{\mathrm{eff}}^{\mathrm{matter}}=2 C \sum_{n=1}^{\infty}\left\{N_{a d j .}^{(+)}\left(I^{(+)}\left[2 a, \beta, z_{\text {adj. }}^{(+)}, n\right]+2 I^{(+)}\left[a, \beta, z_{\text {adj. }}^{(+)}, n\right]+6 I^{(-)}\left[a, \beta, z_{\text {adj. }}^{(+)}, n\right]\right)\right. \\
+N_{\text {adj. }}^{(-)}\left(I^{(-)}\left[2 a, \beta, z_{\text {adj. }}^{(-)}, n\right]+2 I^{(-)}\left[a, \beta, z_{\text {adj. }}^{(-)}, n\right]+6 I^{(+)}\left[a, \beta, z_{\text {adj. }}^{(-)}, n\right]\right) \\
\left.+N_{\text {fnd. }}^{(+)} I^{(+)}\left[a, \beta, z_{\text {fnd. }}^{(+)}, n\right]+N_{\text {fnd. }}^{(-)} I^{(-)}\left[a, \beta, z_{\text {fnd. }}^{(-)}, n\right]\right\}
\end{aligned}
$$

which becomes Eq.(8) in the zero limit of explicit soft scalar masses. Some examples for realizing the suitable dynamical electro-weak symmetry breaking are shown in the following table.

\begin{tabular}{|c|cccc|ccccc||cc|}
\hline & $N_{\text {adj. }}^{(+)}$ & $N_{\text {adj. }}^{(-)}$ & $N_{\text {fnd. }}^{(+)}$ & $N_{\text {fnd. }}^{(-)}$ & $\beta$ & $z_{\text {adj. }}^{(+)}$ & $z_{\text {adj. }}^{(-)}$ & $z_{\text {fnd. }}^{(+)}$ & $z_{\text {fnd. }}^{(-)}$ & $a_{0}$ & $m_{H} / g_{4}^{2}$ \\
\hline \hline$(7)$ & 2 & 0 & 0 & 10 & 0.1 & 0.05 & - & - & 0.05 & 0.0207 & 139 \\
\hline$(8)$ & 2 & 0 & 0 & 6 & 0.15 & 0.1 & - & - & 0.1 & 0.0268 & 139 \\
\hline$(9)$ & 2 & 0 & 0 & 16 & 0.04 & 0 & - & - & 0.03 & 0.0021 & 173 \\
\hline$(10)$ & 2 & 0 & 0 & 4 & 0.07 & 0.5 & - & - & 0.5 & 0.0366 & 138 \\
\hline$(11)$ & 2 & 0 & 0 & 2 & 0.32 & 0 & - & - & 0 & 0.0594 & 135 \\
\hline
\end{tabular}

\section{$5 \quad$ Summary and discussions}

We have studied the Higgs mass in the gauge-Higgs unification theory. Since the Higgs doublet corresponds to the Wilson line phases, it does not have the mass term nor quartic coupling at the tree level. Through the quantum corrections, the Higgs can take a VEV, and its mass is induced. The radiatively induced mass, however, tends to be small, so we lift it to $\mathcal{O}(100) \mathrm{GeV}$ by introducing the $\mathcal{O}(10)$ numbers of bulk fields in the previous works. The perturbation theory can not be reliable when there are a large number of bulk fields.

In this paper we have reanalyzed the Higgs mass and have found that even a small number of bulk field can have the suitable heavy Higgs mass, accompanying the desirable electro-weak symmetry breaking. The expansion formulae for the effective potential are useful to discuss and study analytically the Higgs mass. And we have shown that a small (large) number of bulk fields are enough (needed) when the SUSY breaking mass is large (small). The Higgs mass has the logarithmic dependence on the supersymmetry breaking parameter of the Scherk-Schwarz mechanism. The fine tuning of $\beta$ yields smaller VEV,$a_{0}$, and accordingly enhances the magnitude of the Higgs mass. The analyses in the paper can be applied to the bulk field with an arbitrary representation under an arbitrary gauge group. We have also studied the case of introducing the soft SUSY breaking scalar masses in addition to the SS SUSY breaking. In this case the suitable electro-weak symmetry breaking and the $\mathcal{O}(100)$ $\mathrm{GeV}$ Higgs mass can also be realized by $\mathcal{O}(1)$ numbers of bulk fields. 
Finally, we would like to discuss the higher order operators of Higgs self interactions. We see that the effective potential contains $a^{n}$ interactions by the expansion of the cosine function, which implies $a^{n}=\left(g_{4} R H\right)^{n}$ from Eqs.(11) and (2). When $g_{4} R$ is of order a few $\mathrm{TeV}$, higher order operators, $H^{n}(n \geq 6)$ have the dimensionful suppression of order a few $\mathrm{TeV}$. This means that the contributions from the higher order operators are not so significant.

In connection with new physics expected in the scenario of the gauge-Higgs unification, it may be interesting to comment on the effective 3-point self coupling of $H$ in the models. The coupling is important for the search of the new physics in the future linear colliders 24]. The coupling of the effective $\lambda H^{3}$ interaction is given by $\left.\lambda \equiv \frac{3 g_{4}^{3}}{32 \pi^{6} R} \frac{\partial^{3}(V / C)}{\partial a^{3}}\right|_{a_{0}}$, and the deviation from the tree level SM coupling, $\lambda_{S M}=3 m_{h}^{2} / v$, is estimated by $\Delta \lambda=\left(\lambda-\lambda_{S M}\right) / \lambda_{S M}$ [24]. The value of $\Delta \lambda$ becomes $-17.4 \%$ for the example of $S U(3)_{c} \times S U(3)_{W}$ model $\left(N_{\text {adj. }}^{(+)}=N_{\text {adj. }}^{(-)}=2, N_{\text {fnd. }}^{(-)}=4\right.$, $N_{\text {fnd. }}^{(+)}=0$ with $\left.\beta=0.1\right)$ and $-16.6 \%$ for $S U(6)$ model $\left(N_{\text {adj. }}^{(+)}=2, N_{\text {fnd. }}^{(-)}=10\right.$, $N_{\text {adj. }}^{(-)}=N_{\text {fnd. }}^{(+)}=0$ with $\beta=0.1$ ). As for the examples of section 4 , we show them in the following table.

\begin{tabular}{|c|ccccccccccc|}
\hline & $(1)$ & $(2)$ & $(3)$ & $(4)$ & $(5)$ & $(6)$ & $(7)$ & $(8)$ & $(9)$ & $(10)$ & $(11)$ \\
\hline$\Delta \lambda(\%)$ & -8.6 & -8.3 & -14.0 & -10.2 & -3.1 & -13.7 & -12.0 & -12.0 & -7.6 & -11.2 & -12.7 \\
\hline
\end{tabular}

The effective 3-point self couplings tend to be small comparing to that of the SM. We should notice again that the Higgs field in our model has VEV in $A_{5}$ not $\sigma$. The VEV of $A_{5}$ should be distinguished from that of $\sigma$ in the dynamically induced effective potential in the gauge-Higgs unification theory[20]. Since $H$ is the field of the $D$-flat direction, which is massless at tree level, it corresponds to the lighter Higgs scalar in the MSSM, $h^{0}$. Since $h^{0}$ becomes the SM-like Higgs in the large soft SUSY breaking masses, we have compared the effective 3-point self coupling to the SM one in the above estimation of $\Delta \lambda$. Other masses of Higgs eigenstates, charged Higgs, neutral scalar, and heavier pseudo-scalar, can be calculated by the effective potential of these directions 25].

\section{Acknowledgements}

We would like to thank M. Tanabashi and Y. Okada for useful discussions which become one of the motivations of this work. We would like to thank N. Okada for a lot of useful and helpful discussions. K.T. would thank the colleagues in Osaka University and gives special thanks to the professor Y. Hosotani for valuable discussion. K.T. is supported by the 21st Century COE Program at Osaka University. T.Y. would like to thank the Japan Society for the Promotion of Science for financial support. N.H. is supported in part by Scientific Grants from the Ministry of Education and Science, Grant No. 14740164, No. 16028214, and No. 16540258. 


\section{References}

[1] N. S. Manton, Nucl. Phys. B 158, (1979), 141;

D. B. Fairlie, J. Phys. G 5, (1979), L55; Phys. Lett. B 82, (1979), 97.

[2] Y. Hosotani, Phys. Lett. B126 (1983), 309; Ann. of Phys. 190 (1989), 233; Phys. Lett. B129 (1984), 193; Phys. Rev. D29 (1984), 731.

[3] N. V. Krasnikov, Phys. Lett. B 273, (1991), 246;

H. Hatanaka, T. Inami and C. S. Lim, Mod. Phys. Lett. A 13, (1998), 2601;

G. R. Dvali, S. Randjbar-Daemi and R. Tabbash, Phys. Rev. D 65, (2002), 064021;

N. Arkani-Hamed, A. G. Cohen and H. Georgi, Phys. Lett. B 513, (2001), 232;

I. Antoniadis, K. Benakli and M. Quiros, New J. Phys. 3, (2001), 20.

[4] M. Kubo, C. S. Lim and H. Yamashita, Mod. Phys. Lett. A 17 (2002), 2249.

[5] L. J. Hall, Y. Nomura and D. R. Smith, Nucl. Phys. B 639 (2002), 307.

[6] G. Burdman and Y. Nomura, Nucl. Phys. B 656 (2003), 3.

[7] N. Haba and Y. Shimizu, Phys. Rev. D 67 (2003), 095001;

I. Gogoladze, Y. Mimura and S. Nandi, Phys. Lett. B 560 (2003), 204; Phys. Lett. B 562 (2003), 307.

[8] C. A. Scrucca, M. Serone and L. Silvestrini, Nucl. Phys. B 669 (2003), 128.

[9] C. Csaki, C. Grojean, H. Murayama, L. Pilo and J. Terning, Phys. Rev. D 69 (2004), 055006 .

[10] I. Gogoladze, Y. Mimura, S. Nandi and K. Tobe, Phys. Lett. B 575 (2003), 66;

K. Choi, N. Haba, K. S. Jeong, K. i. Okumura, Y. Shimizu and M. Yamaguchi, JHEP 0402 (2004), 037.

[11] N. Haba, Y. Hosotani, Y. Kawamura and T. Yamashita, Phys. Rev. D 70 (2004), 015010 .

[12] N. Haba and T. Yamashita, JHEP 0402 (2004), 059; JHEP 0404 (2004), 016.

[13] Y. Hosotani, S. Noda and K. Takenaga, Phys. Rev. D 69 (2004), 125014; Phys. Lett. B 607 (2005), 276.

[14] S. Coleman and E. Weinberg, Phys. Rev. D 7 (1973), 1888.

[15] J. Scherk and J. H. Schwarz, Phys. Lett. B 82 (1979), 60; Nucl. Phys. B 153 (1979), 61.

[16] P. Fayet, Phys. Lett. B 159 (1985), 121; Nucl. Phys. B 263 (1986), 649.

[17] K. Takenaga, Phys. Lett. B 425 (1998), 114; Phys. Rev. D 58 (1998), 026004.

[18] I. Antoniadis, Phys. Lett. B246 (1990), 377;

I. Antoniadis, C. Munoz, and M. Quiros, Nucl. Phys. B397 (1993), 515;

A. Pomarol and M. Quiros, Phys. Lett. B 438 (1998), 255;

I. Antoniadis, S. Dimopoulos, A. Pomarol and M. Quiros, Nucl. Phys. B 544 (1999), 503 ;

A. Delgado, A. Pomarol and M. Quiros, Phys. Rev. D 60 (1999), 095008.

[19] G.V. Gersdorff, M. Quiros and A. Riotto, Nucl. Phys. B 634 (2002), 90;

G.V. Gersdorff and M. Quiros, Phys. Rev. D 65 (2002), 064016.

[20] N. Haba, K. Takenaga and T. Yamashita, hep-th/0411113, to appear in Phys.Rev.D.

[21] N. Haba and T. Yamashita, Phys. Lett. B 597 (2004), 166.

[22] K. Takenaga, Phys. Lett. B 570 (2003), 244. 
[23] N. Haba, K. Takenaga and T. Yamashita, hep-th/0410244, to appear in Phys.Lett.B.

[24] S. Kanemura, S. Kiyoura, Y. Okada, E. Senaha and C. P. Yuan, Phys. Lett. B 558 (2003), 157;

S. Kanemura, Y. Okada, E. Senaha and C. P. Yuan, hep-ph/0408364.

[25] N. Haba, K. Takenaga and T. Yamashita, in preparation. 